\section{Novelle des Arzneimittelgesetzes}

\section{Auswirkungen auf die Allergiediagnostik}

\begin{abstract}
Mit Inkrafttreten der 15. Novelle des Arzneimittelgesetzes müssen allergologisch tätige Ärzte bei der zuständigen Behörde anzeigen, wenn sie mit patienteneigenem Material Testungen vornehmen, und jederzeit mit Inspektionen rechnen. Auch wenn nun ein Mehraufwand droht, wäre es unvernünftig, zukünftig auf diese Allergietestungen zu verzichten.
\end{abstract}

T estsubstanzen für den Epikutantest oder den Pricktest sind Arzneimittel im Sinne des $\$ 2$ Abs. 1 des Arzneimittelgesetzes (AMG). Definitionsgemäß trifft dies auch für Testzubereitungen mit patienteneigenem Material wie Körperpflegeprodukte oder Berufsstoffe für den Epikutantest oder Nahrungsmittel für den Reib-, Scratch- oder Pricktest zu. Bisher waren jedoch solche vom Arzt oder unter seiner unmittelbaren fachlichen Verantwortung individuell für einen Patienten hergestellten Zubereitungen gemäß \$4a AMG vom Anwendungsbereich des AMG ausgenommen. Dies hat sich seit diesem Sommer geändert. Mit Inkrafttreten der 15. AMGNovelle (Gesetz zur Änderung arzneimittelrechtlicher und anderer Vorschriften, BGBl Teil I, Nr. 43, S. 1990) am 23.7.2009 unterliegen Ärzte sowie andere Personen, die zur Ausübung der Heilkunde befugt sind, und die Arzneimittel zur Anwendung bei ihren Patienten herstellen, dem Anwendungsbereich des AMG und damit der Überwachung nach $\$ 64$ AMG. Sie benötigen dafür allerdings keine Herstellungserlaubnis ( $\$ 13$ Abs. 2b AMG).

\section{Bedeutung für die} allergologische Praxis

1. Anzeigepflicht: Wer weiterhin Epikutan-, Reib-, Scratch- oder Pricktestungen mit patienteneigenem Material vornehmen will, muss dies der zuständigen Behörde gemäß \$67 AMG anzeigen. Die
Zuständigkeiten sind in den einzelnen Bundesländern unterschiedlich geregelt - so sind in Niedersachsen die Staatlichen Gewerbeaufsichtsämter zuständig, in $\mathrm{Ba}$ yern die Bezirksregierungen. Für die Anzeige nach $\$ 67$ AMG gibt es eine Übergangsfrist bis zum 1. Februar 2010 (\$144 Abs. 7 AMG). Wer danach die Anzeige unterlässt, begeht eine Ordnungswidrigkeit. Nach ersten Informationen kann die Anzeige als formloses Anschreiben mit summarischer Beschreibung der durchgeführten Testungen an die zuständige Behörde erfolgen. Es wird also wohl nicht erforderlich sein, dass jede einzelne Testung anzuzeigen ist.

2. Überwachung: Gemäß $\$ 64$ Abs.1 AMG unterliegen alle anzeigenden Einrichtungen und Betriebe der Überwachung durch die zuständige Behörde. Gemäß $\$ 64$ Abs.3 AMG muss mit behördlichen Inspektionen gerechnet werden. $\mathrm{Ob}$ eine Inspektion angekündigt wird oder nicht, liegt im Ermessen der zuständigen Behörde. Zu den Einzelheiten bezüglich des Prüfumfangs wird es sicherlich noch eine Absprache mit allen in Deutschland beteiligten Überwachungsbehörden geben, damit die Überwachung weitgehend einheitlich stattfindet.

In erster Linie wird es aber wichtig sein, dass die Arzneimittel nach dem aktuellen Stand von Wissenschaft und Technik hergestellt werden, und dies entsprechend dokumentiert wird. Hierzu sind Arbeitsanweisungen (,standard operating proce- dures"; SOP) hilfreich. Für die Epikutantestung können entsprechende Vorlagen, die man bei Bedarf individuell anpassen kann, und weitere hilfreiche Unterlagen auf der Website www.hautstadt.de heruntergeladen werden. Nach dem Login findet man die Texte im „Infozentrum Allergie“ unter „Qualitätsmanagement".

\section{Warum sich die Mühe lohnt}

Die individuelle Testung ist ein unverzichtbarer Bestandteil der allergologischen Versorgung unserer Patienten. Wenn man die allergologische Diagnostik aufTestungen mit zugelassenen, kommerziell erhältlichen Testsubstanzen beschränkt, werden sicher relevante Sensibilisierungen übersehen. Um Nahrungsmittelunverträglichkeiten und Arzneimittelreaktionen aufzuklären ist die Bedeutung der individuellen Testung evident. Aber auch bei einer Kontaktallergie ist die Testung mit patienteneigenem Material erforderlich. Die Epikutantest-Programme der etablierten Hersteller können gar nicht das breite Spektrum potenzieller Allergene voll abdecken, und sie können ebenso wenig stets auf dem Stand der aktuellen AllergenExposition der Patienten sein.

Wenn allergologisch tätige Ärzte die Mühen des neuen AMG nicht auf sich nehmen wollen und auf solche Testungen verzichten, bedeutet das in der Kontaktallergie, dass viele Sensibilisierungen nicht mehr diagnostiziert werden. Man wird lediglich noch registrieren, dass der Patient ein bestimmtes Produkt nicht vertragen hat. Die Folge für den betroffenen Patienten ist, dass er „sein“ Allergen nicht kennt, und daher bei der Suche nach einer Alternative an ein Produkt mit demselben allergenen Inhaltstoff geraten kann, mit der Folge eines Rezidives des allergischen Kontaktekzems. Über den individuellen Fall hinaus würde durch die Nicht-Testung auch die Möglichkeit behindert, neue, bisher nicht systematisch untersuchte Allergene zu erkennen.

PD Dr. med. Johannes Geier

Vorsitzender der Deutschen

Kontaktallergie-Gruppe (DKG)

Universitäts-Hautklinik Göttingen

von-Siebold-Str. 3

37075 Göttingen

E-Mail:jgeier@ivdk.org 